\title{
French survey on a cohort of emergency general surgery modifications induced by lockdown of the SARS-CoV-2 pandemic
}

\author{
Clement Julien $^{1,2}$ (D) Pauline Duconseil ${ }^{1} \cdot$ Diane Mege $^{3} \cdot$ Damien Massalou $^{4} \cdot$ Todor Ivanov $^{5} \cdot$ Raffaele Brustia $^{5} \cdot$ \\ Ghislain Pauleau $^{6}$. Stéphane V. Berdah ${ }^{1} \cdot$ Thierry Bège $^{1} \cdot$ Collaborators' Working Group $^{\prime}$
}

Received: 27 October 2020 / Accepted: 3 December 2020 / Published online: 15 January 2021

(C) Royal Academy of Medicine in Ireland 2021

\begin{abstract}
Purpose The brutal COVID-19 pandemic has majorly impacted populations and health systems, and surgeons have observed dramatic changes in their daily clinical activities. A survey of French digestive surgeons was conducted to assess these changes. Methods An electronic survey was sent to French digestive and general surgeons in the Societe Francaise de Chirurgie Digestive (SFCD) to assess the surgeons' daily activity during the pandemic and investigate changes in patients' management. The care deviations were classified as delay of management, modification of strategy, or modification of organization, and the impact of these changes on patients was evaluated by the surgeon's estimation of loss of chance.

Results A major reduction in surgical elective activity was observed in 50 (75\%) of the 67 hospitals that responded. Of these, 48 hospitals $(71.6 \%)$ reported receiving SARS-CoV-2 patients. A deviation from usual care was observed in $10 \%$ of patients admitted for emergency general surgery. Among 140 patients presenting a deviation from usual care, 74 (52.9\%) had delayed management, $53(37.9 \%)$ had a modification of strategy, and $64(45.7 \%)$ had a modification of organization. Medical treatment instead of surgical treatment was decided for 37 (26.4\%) patients, resulting in a high loss of chance for 6 patients. Delays $(p<$ $0.001)$ and a switch from surgical to medical treatment $(p=0.002)$ were independently correlated with overall loss of chance based on multivariate analysis.

Conclusion This study highlighted the deviations in general emergency surgery patients and provided implications for the solutions that should be implemented during a new health crisis.
\end{abstract}

Keywords Acute abdominal surgery $\cdot$ COVID-19 $\cdot$ Digestive surgery $\cdot$ Loss of chance $\cdot$ SARS-CoV-2

\section{Introduction}

Over a few weeks, SARS-CoV-2, an emerging disease localized to a region of China, became a global pandemic [1]. The brutality of this pandemic and the large number of patients suffering from severe forms of the disease has had a major

No previous communication to a society or meeting

Clement Julien

clement.julien@ap-hm.fr

1 Aix-Marseille University, Assistance Publique Hôpitaux de Marseille, Department of Gastrointestinal Surgery, Nord University Hospital, Marseille, France

2 Department of General Surgery, Sainte-Anne Military Teaching Hospital, Toulon, France

3 Aix-Marseille University, Assistance Publique Hôpitaux de Marseille, Department of Digestive Surgery, Timone University Hospital, Marseille, France impact on populations and health systems, regardless of health facilities and geographical location [2]. France was one of the earliest and most affected countries in the world, and its national health system was severely strained while undergoing deep and rapid reorganization to face this unprecedented crisis.

4 Acute Care Surgery, Chirurgie Générale d'Urgence University Hospital of Nice, Hôpital Pasteur 2, Côte d'Azur University, 30 voie romaine, 06000 Nice, France

5 Assistance Publique-Hôpitaux de Paris-Department of Digestive and Hepato-pancreatic-biliary Surgery, Hôpital Henri-Mondor, 94010 Creteil, France

6 Department of Gastrointestinal Surgery, Laveran Military Teaching Hospital, Marseille, France 
The French national lockdown recommended home confinement beginning March 17, 2020, shutting schools, forcing the population to work from home, and restricting movement with minor exceptions for essential needs [3]. The population was urged not to overload hospital services, and concern was communicated that they could be contaminated in emergency departments overwhelmed by cases of COVID- 19 . Additionally, emergency care pathways were dedicated to treating COVID-19, providing screening by polymerase chain reaction (PCR) tests and/or thoracic computed tomography (CT). This situation may have increased the time between admission and surgical care (door-to-surgery time). Surgeons also sustained radical changes in their practices $[4$, $5]$ through massive deprogramming of non-emergency procedures to decrease disease transmission and spare anesthesia drugs and personal protective equipment [6]. These changes decreased the number of patients consulting for abdominal pain and digestive emergencies and resulted in severe clinical presentations $[7,8]$. This survey aimed to assess changes in emergency surgical practice within the community of French digestive surgeons critically.

\section{Materials and methods}

\section{Study design}

The survey was designed with two separate questionnaires that were sent to the community of French digestive and general surgeons in the Societe Francaise de Chirurgie Digestive (SFCD). The questionnaires were both concerned with the French lockdown from March 17 to May 11, 2020 (which corresponded to the study period). The first generic e-survey focused on daily surgical activities during the pandemic, including the type of facility, daily presence of the surgeon, reduction of surgical activity, care of COVID-19 patients, participation in non-surgical COVID-19 care, and use of teleconsultation. The geographic area of each facility was considered as exposed to high or low pandemic pressure according to the public authorities' definition (considering the circulation rate of the virus and the number of hospitalized patients) [9]. The second questionnaire investigated changes in patients' management. For each patient with a deviation in care during the period, an anonymous questionnaire was filled out with the following items: age, gender, ASA score [10], COVID-19 status (positive, negative, unknown), diagnosis, date of admission (for ease of analysis, a three-level categorical variable "confinement period" was created), surgical procedure, postoperative complications (according to the DindoClavien classification [11]), and length of hospital stay. The centers were also asked to report the total number of patients admitted for acute abdominal care during the same period.

\section{Variable definitions}

Deviation from standard care was classified into three categories, and each patient could fulfill one to three categories. The categories were as follows:

1. Delay of management was considered as a deviation when the date of admission was intentionally delayed from the onset of symptoms. Reasons for this delay could include the patient's fear of being hospitalized or a practitioner's advice (general practitioner [GP] or surgeon) against early admission. The duration was quantified in days and was considered to be major when $\geq 5$ days.

2. Modification of treatment strategy included any change in treatment class (surgical to medical or the reverse), surgical procedure (laparoscopic to open approach, no drain, damage control, etc.), degree of emergency (e.g., patients requiring emergency surgery for a canceled elective procedure), and access to an intensive care unit (ICU; i.e., non-admission to an ICU for monitoring because of limited access).

3. Modification of logistical organization concerned all inter-facility transfers, modification of the usual admission mode, implementation of specific prevention procedures in the operating room for patients with a suspected COVID-19 infection, and any delay within the hospital (e.g., the supplementary time required for SARS-CoV-2 diagnostic procedure with CT-thorax and PCR sampling).

Each surgeon was required to estimate the impact of these deviations on the patients' loss of chance (LOC) using a subjective scale (no loss, moderate, or high). The overall LOC was considered moderate to high.

Statistical analyses were performed using IBM SPSS 23.0 (IBM Inc., New York, NY, USA). Categorical variables were described in terms of frequency (percentages), and continuous variables were described as mean \pm range (SD). Univariate analyses were conducted using a Student's $t$ test or the Mann-Whitney test for continuous variables and the chisquare test or Fisher's exact test for categorical variables. A two-tailed $p$ value $\leq 0.05$ was considered statistically significant. Multivariate analyses were performed using a backward stepwise logistic regression model adjusted for covariates significant at $p \leq 0.1$ based on univariate analysis.

\section{Results}

\section{Impact on health centers' surgical activity}

Responses concerning changes in global surgical activities were obtained from 67 hospitals (Table 1). The responders were mostly located in general hospitals ( $n=47,70.1 \%$ ) from 
Table 1 Impact on surgeons' hospital activity

\begin{tabular}{|c|c|c|c|}
\hline \multirow[b]{2}{*}{ Characteristics } & \multicolumn{3}{|c|}{ According to the hospital area } \\
\hline & $\begin{array}{l}\text { High pressure } \\
n=18(\%)\end{array}$ & $\begin{array}{l}\text { Low pressure } \\
n=49(\%)\end{array}$ & $\begin{array}{l}\text { Univariate } \\
p \text { value }\end{array}$ \\
\hline Type of hospital: & & & 0.58 \\
\hline General hospital & $14(77.8)$ & $33(67.3)$ & \\
\hline Regional/University Hospital & $2(11.1)$ & $3(6.1)$ & \\
\hline Private hospital & $2(11.1)$ & $13(26.6)$ & \\
\hline Reduction of surgical activity: & & & 0.63 \\
\hline$>75 \%$ & $15(83.3)$ & $35(71.4)$ & \\
\hline $50-75 \%$ & $3(16.7)$ & $10(20.4)$ & \\
\hline $25-50 \%$ & $0(0)$ & $4(8.2)$ & \\
\hline$<25 \%$ & $0(0)$ & $0(0)$ & \\
\hline Reduction of surgeon's daily presence & & & 0.76 \\
\hline $75 \%$ & $2(11.1)$ & $7(14.3)$ & \\
\hline $50-75 \%$ & $5(27.8)$ & $13(26.5)$ & \\
\hline $25-50 \%$ & $7(38.9)$ & $13(26.5)$ & \\
\hline$<25 \%$ & $4(22.2) \mathrm{d}$ & $16(32.7)$ & \\
\hline Surgical activity in another hospital: & & & 0.71 \\
\hline No & $13(72.2)$ & $39(79.6)$ & 0.52 \\
\hline Yes, Public structure & $3(16.7)$ & $5(10.2)$ & \\
\hline Yes, Private structure & $2(11.1)$ & $5(10.2)$ & \\
\hline \multicolumn{4}{|l|}{ Management of SARS-CoV-2 patient: } \\
\hline In the surgical unit & $16(88.9)$ & $32(65.3)$ & 0.07 \\
\hline Operated & $15(83.3)$ & $26(53.1)$ & 0.03 \\
\hline \multicolumn{4}{|l|}{ Modification of surgeon's activity: } \\
\hline Participation to a COVID unit & $9(50.0)$ & $13(26.5)$ & 0.07 \\
\hline Participation to ICU & $5(27.8)$ & $8(16.3)$ & 0.29 \\
\hline Participation to emergency unit & $9(50.0)$ & $32(65.3)$ & 0.25 \\
\hline Participation to hospital reorganization & $10(55.6)$ & $22(44.9)$ & 0.44 \\
\hline No participation & $1(5.6)$ & $4(8.2)$ & 1 \\
\hline Use of teleconsultation: & $11(61.1)$ & $34(69.4)$ & 0.52 \\
\hline
\end{tabular}

low-pressure areas. A reduction in surgical elective activity greater than $75 \%$ was observed in 50 centers $(74.6 \%$ of responders), and significantly more COVID-19 patients underwent surgery in high-pressure areas compared with low-pressure areas $(83 \%$ vs. $53 \%, p=0.03)$. The daily presence of surgeons varied widely across the centers, with $22.3 \%$ $(n=15)$ of them performing surgical procedures in another facility. Of the surgeons, $48(71.6 \%)$ reported receiving COVID-19 patients in their surgical unit, and $41(61.2 \%)$ operated on infected patients. Additionally, 45 (67.2\%) turned their consultations into teleconsultations. Most of them partially shifted their daily activity toward non-surgical management of COVID-19 patients, as follows: $22(32.8 \%)$ in COVID units, $13(19.4 \%)$ in an ICU, $41(61.2 \%)$ in emergency units, and $32(47.8 \%)$ in the management of hospital reorganization.

\section{Impact on patient management}

Six centers reported the total number of patients admitted for digestive emergencies during this period, and a deviation from usual care was observed in $10 \%$ of them (53 out of 532). The other centers reported deviations without providing the total number of patients admitted. In total, the description of deviations concerned 140 patients. The patients' characteristics are reported in Table 2.

In the centers, 11 patients $(7.8 \%)$ were positive for COVID-19. The three most common abdominal diseases were appendicitis $(n=34,24.3 \%)$, occlusion $(n=32,22.9 \%)$, and biliary disease $(n=27,19.3 \%)$. Of the patients, $96(68.6 \%)$ patients required surgery, and the surgeries for 25 of them (17.9\%) included complementary protection for SARS-CoV2. Twenty-seven $(19.3 \%)$ patients presented severe 
Table 2 Patients characteristics

\begin{tabular}{ll}
\hline Characteristics & $n=140(\%)$ \\
\hline General/regional hospital & $98 / 42$ \\
Date of admission: & \\
March 17th-31st: beginning of quarantine & $37(26.4)$ \\
April 1st-15rd & $48(34.3)$ \\
April 16th-May 11th: end of quarantine & $55(39.3)$ \\
Age, years ( \pm SD) & $59(22)$ \\
Gender, male & $88(62.9)$ \\
Region with high pressure & $43(30.7)$ \\
ASA score: 1-2 & $103(73.6)$ \\
COVID status: & \\
Positive & $11(7.8)$ \\
Negative & $95(67.9)$ \\
Unknown & $34(24.3)$ \\
Diagnosis: & \\
Appendicitis & $34(24.3)$ \\
Occlusion & $32(22.9)$ \\
Biliary disease & $27(19.3)$ \\
Proctology/abscess & $15(10.7)$ \\
Complicated cancer & $7(5.0)$ \\
Peritonitis & $7(5.0)$ \\
Mesenteric ischemia & $4(2.8)$ \\
Colitis/diverticulitis & $3(2.1)$ \\
Other & $11(7.9)$ \\
Need for Surgical procedure & $96(68.6)$ \\
Postoperative complications: & \\
No complication & $27(19.3)$ \\
Clavien 1-2 & $8(7)$ \\
Clavien $\geq 3$ & \\
Duration of hospital stay ( \pm SD) & \\
\hline & \\
&
\end{tabular}

complications (Clavien $\geq 3$ ), including 7 (5.0\%) deaths during hospitalization.

\section{Deviation from standard care}

The deviations from standard care were classified into three categories: delay of management, modification of treatment strategy, and modification of organization (Fig. 1). Of the patients, 33 presented two or three deviations. The characteristics of the patients according to the type of deviation are shown in Table 3.

1. Delay of management was the most common reported deviation and concerned 74 (52.9\%) patients. Among 40 patients $(28.6 \%)$ who waited for 5 days or more, 19 had received previous consultation from a GP, while 21 had not consulted at all. This led to a high LOC of 2 and 6 , respectively. Among 34 patients (24.3\%) who waited for
4 days or less, 12 had received previous consultation from a GP, while 22 had not consulted at all, leading to a high LOC of 0 and 3, respectively.

2. Concerning the modification of strategy, medical treatment instead of surgical treatment was decided for 37 (26.4\%) patients. Of these patients, 12 eventually underwent surgery. The two pathologies most frequently affected by this modification were cholecystitis $(n=15)$ and appendicitis $(n=10)$. This generated no LOC for 3 patients (one biliary disease, one appendicitis, and one digestive occlusion) but a high LOC for 6 patients (two biliary diseases, two occlusions, one mesenteric ischemia, and one Fournier's gangrene). In the subgroup of patients affected by biliary disease and treated by medical instead of surgical treatment, 2 died, 4 were re-hospitalized for medical treatment failure, and 3 underwent surgery. The medical treatment of appendicitis resulted in no LOC for 1 patient and moderate LOC for 9 patients; 4 patients eventually underwent surgery. Seven $(5.0 \%)$ patients underwent surgery after a canceled procedure, including 4 patients with biliary disease.

3. Regarding management of organization, 24 (17.1\%) patients were impacted by a delay within the hospital, and $25(17.9 \%)$ were operated on with complementary protection for COVID-19. The median delay within the hospital was $9 \mathrm{~h}$ (range 2-72). The most common reason for this delay was waiting for the patient's COVID-19 status results. Furthermore, $19(13.6 \%)$ patients were transferred to another hospital because of limited access to the ICU or upgraded medical technical platforms. The patients who suffered the most from organizational deviations had occlusion $(n=17)$ or appendicitis $(n=17)$. Among the patients with occlusion, 2 died, 2 were transferred to the ICU, and another one had no access to the ICU even when admission to the ICU was indicated. The treatment of occlusion for 13 patients was surgery, including 6 patients who received complementary protection for COVID-19. Among the 17 patients with appendicitis, 2 (11.8\%) developed severe complications (Clavien $\geq 3$ ), 10 were operated on with complementary protection for COVID-19, and 3 were transferred to another hospital.

\section{Loss of chance}

The surgeons estimated that the deviations from usual care were responsible for an overall LOC in $80 \%$ of patients (moderate LOC in 82 patients [58.6\%] and high LOC in 30 patients [21.4\%]). Only 28 patients (20\%) had no consequence from the deviations. Moderate LOC was significantly associated with the patient's age $(p=0.002)$, intentional delay before consultation $(p=0.001)$, switch from surgical treatment to 
Table 3 Deviation from usual care

\begin{tabular}{|c|c|c|c|c|}
\hline Type of deviation & $\begin{array}{l}\text { All } \\
n=140(\%)\end{array}$ & $\begin{array}{l}\text { No loss of chance } \\
n=28(\%)\end{array}$ & $\begin{array}{l}\text { Loss of chance } \\
n=112\end{array}$ & $\begin{array}{l}\text { Univariate } \\
p \text { value }\end{array}$ \\
\hline \multicolumn{5}{|l|}{ Delay } \\
\hline Intentional delay before consultation & $74(52.9)$ & $8(28.6)$ & $66(58.9)$ & 0.004 \\
\hline Delay $>5$ days & $40(28.6)$ & $4(14.3)$ & $36(32.1)$ & 0.07 \\
\hline Previous consultation by a general practitioner & $25(17.9)$ & $3(10.7)$ & $22(19.6)$ & 0.41 \\
\hline \multicolumn{5}{|l|}{ Strategy } \\
\hline \multicolumn{5}{|l|}{ Change in therapeutic strategy: } \\
\hline Surgical switch to medical & $37(26.4)$ & $3(10.7)$ & $34(30.4)$ & 0.05 \\
\hline Medical switch to surgical & $0(0)$ & $0(0)$ & $0(0)$ & 1 \\
\hline Cancelled elective procedure operated in emergency & $7(5.0)$ & $1(3.6)$ & $6(5.4)$ & 1 \\
\hline Limited access to ICU & $5(3.6)$ & $0(0)$ & $5(4.5)$ & 0.58 \\
\hline \multicolumn{5}{|l|}{ Change in surgical procedure: } \\
\hline Laparoscopy to laparotomy & $3(2.1)$ & $0(0)$ & $3(3.7)$ & 1 \\
\hline Other change (open abdomen...) & $5(3.6)$ & $0(0)$ & $5(4.5)$ & 0.58 \\
\hline \multicolumn{5}{|l|}{ Organization } \\
\hline Delay within the hospital & $24(17.1)$ & $5(17.9)$ & $19(17.0)$ & 1 \\
\hline Complemented protection for SARS-CoV-2 at the operating theatre & $25(17.9)$ & $9(32.1)$ & $16(14.3)$ & 0.03 \\
\hline Transfer in other hospital & $19(13.6)$ & $5(17.9)$ & $14(12.5)$ & 0.54 \\
\hline Modification of admission mode & $9(6.4)$ & $1(3.6)$ & $8(7.1)$ & 0.69 \\
\hline
\end{tabular}

$I C U$ intensive care unit

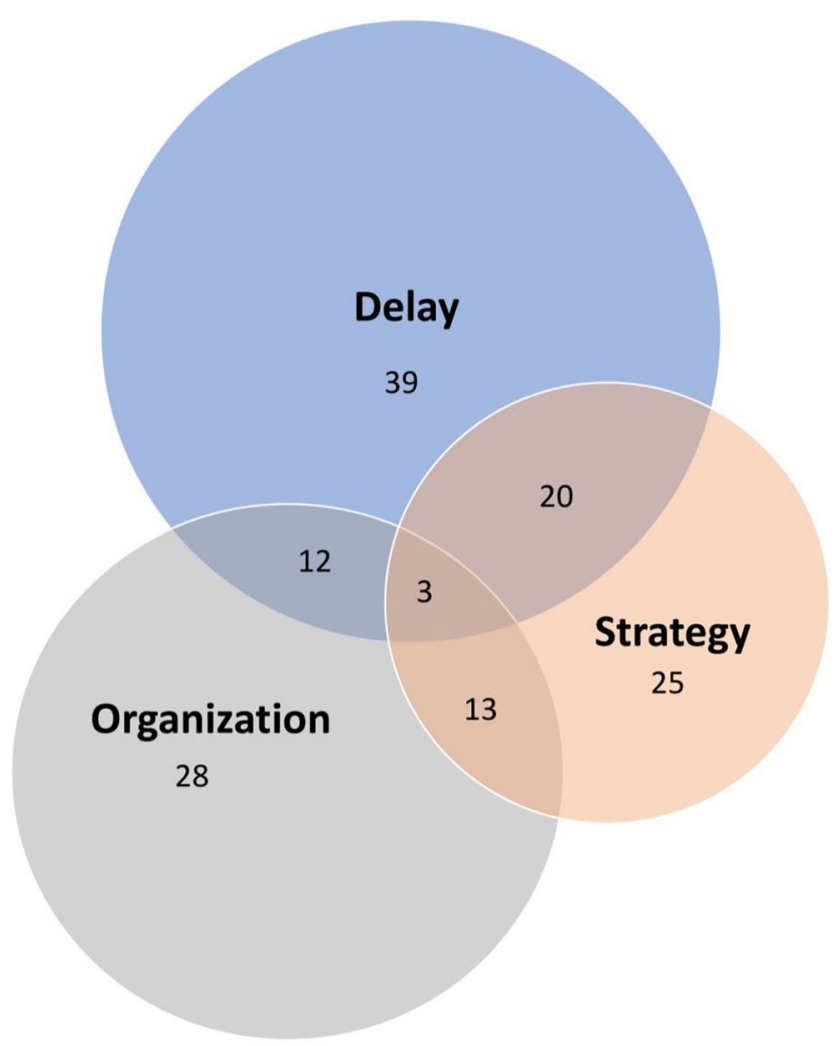

Fig. 1 Venn diagram representing the three different deviations. The wideness of the circles illustrates the number of events for each type of deviation. The circles cross when patients experienced two or more types of deviations. medical treatment $(p=0.027)$, and complementary protection for SARS-CoV-2 during surgery $(p=0.027)$. High LOC was significantly associated with the patient's age $(p=0.002)$, ASA score $(p=0.001)$, the type of pathology $(p=0.002)$, and being in a high-pressure area $(p=0.014)$. Peritonitis and mesenteric ischemia were more often associated with high LOC: $0 \%$ and $0 \%$ versus $13.3 \%$ and $10.0 \%$, respectively. Considering overall LOC, intentional delay before consultation $(p=0.004)$ and complementary protection for SARSCoV-2 during surgery $(p=0.027)$ showed a statistical significance. In the multivariate analysis, variables that independently correlated with overall LOC were an ASA score of $1-2(p=0.014, \mathrm{OR}=0.21,95 \%$ IC $0.06-0.73)$, medical treatment instead of surgical treatment $(p=0.002, \mathrm{OR}=$ 9.14, 95\% IC 2.29-36.49), and intentional delay before consultation $(p<0.001, \mathrm{OR}=6.59,95 \%$ IC $2.40-18.14)$.

\section{Discussion}

The brutal COVID-19 pandemic saturated and disorganized French hospitals. Non-emergency surgeries were canceled, and the number of consultations dramatically decreased [4, 6]. For these reasons, surgeons' daily activity was modified, with a reduction in surgical elective activity greater than $75 \%$ for $74.6 \%$ of hospitals. Teleconsultation was used as an adaptation to ensure continuity of care; it was decided on and applied by most hospitals (67.2\%). 
Access to care was maintained, but patients' fear of contracting COVID-19 and the saturation of hospitals and their reorganization induced deviations from usual care [7, 12, 13]. This study highlighted this fact by identifying 140 deviations from usual care classified as delay in consultation $(52.9 \%)$, change in therapeutic strategy, and modified organization. To measure the effect of these deviations on patients' outcomes, the LOC, which is subjective but relevant for surgeons, was used. Three independent factors predicting LOC were identified: an ASA score of 3-4, medical treatment instead of surgical treatment, and intentional delay before consultation. Organization deviation did not appear to have an impact on the severity of complications or LOC for patients.

As expected, fragile patients with serious abdominal pathologies (e.g., mesenteric ischemia, peritonitis) were more impacted by any deviation than younger patients with deferrable emergencies, such as appendicitis or proctologic diseases. Intentional delay before consultation was an expected pejorative factor since acute abdominal pathology outcomes are conditioned by the delay of management. However, a previous consultation by a GP limited its impact through a better selection and orientation of the patients. The change in therapeutic strategy was a one-way change, and some patients received medical treatment instead of an indicated surgery to spare them from complications, COVID infection, transfer to ICU, or a longer stay. Successes in the medical treatment of appendicitis were observed, but patients with biliary diseases experienced more medical treatment failures and thus more LOC. Therefore, surgeons should be cautious when making this decision, as delayed surgery in case of failure can be deleterious and represent a real LOC.

During national lockdown, each country witnessed a brutal drop in emergency department visits of at least $25 \%$ of usual activity[14]. Moreover, Patriti et al. described a decrease in surgical emergencies $[12,15]$. Some patients were able to be treated and monitored by their GP at home, but late admission was probably responsible for an ICU transfer for 3 patients [15].

Ignorance of certain modes of transmission routes or symptoms of SARS-CoV-2 was also responsible for surgical changes. Indeed, COVID-19 digestive symptoms complicated diagnoses $[16,17]$. Moreover, the virus has a fecal tropism that may be responsible for contamination during exposure $[18,19]$, and additional protective measures had to be established in operating theaters in case of an infected patient [20]. These measures were observed 25 times in this study. Furthermore, the use of laparoscopy has been contraindicated during the pandemic because of the potential vaporization of the virus, especially during gas exsufflation $[21,22]$; in the present study, 3 patients were preferably operated by laparotomy for this reason. These novel specificities complicated the management and circuits of infected and non-infected patients.
This work analyzed the deviations in usual care to improve the management of patients in case of a second wave. Patients should be reassured about the continuity of care and hospital safety, should be informed of excess loss of chance and morbidity if waiting to consult, and should have ease of medical access. The possible response to a long-term disaster is telehealth visits, whose access must be extended [15]. Moreover, the delay within hospitals must be improved. Hospitals need to adapt to increasing patient flow, and serious situations need to be identified early. Therefore, surgeons must be involved in reorganization and triage. Additionally, clinicians should be cautious when changing therapeutic strategy, especially for biliary disease.

This work has several limitations: (i) unequal response rate of centers (high vs. low pressure, and university vs. general and private hospitals) leading to under-estimation; (ii) no comparison to the usual situation limit extrapolation; (iii) no normal consultation time for abdominal pain, which is a bias for delay deviation; and (iv) the subjective nature of LOC, which nonetheless seemed to reflect the real situation.

\section{Conclusion}

This work showed an excellent portrayal of usual surgical activity and emergency management. The major deviations to usual care were identified, and the necessary adaptations were proposed. Patients must be reassured of the safety of disaster management to limit delays in the management of serious pathologies, and clinicians should carefully select management strategy changes, especially for biliary disease.

Acknowledgments SFCD: Société Française de Chirurgie Digestive

\section{References}

1. Zhu N, Zhang D et al (2020) A novel coronavirus from patients with pneumonia in China, 2019. N Engl J Med 382:727-733. https://doi.org/10.1056/NEJMoa2001017

2. Spinelli A, Pellino G (2020) COVID-19 pandemic: perspectives on an unfolding crisis. Br J Surg 107:785-787. https://doi.org/10. $1002 /$ bjs. 11627

3. https://www.gouvernement.fr/info-coronavirus/les-actions-dugouvernement

4. Cano-Valderrama O, Morales X, Ferrigni CJ et al (2020) Reduction in emergency surgery activity during COVID-19 pandemic in three Spanish hospitals: reduction in emergency surgery during COVID19. Br J Surg. 107:e239. https://doi.org/10.1002/bjs.11667

5. Zheng MH, Boni L, Fingerhut A (2020) Minimally invasive surgery and the novel coronavirus outbreak: lessons learneD in China and Italy. Ann Surg Publish Ahead of Print 272:e5-e6. https://doi. org/10.1097/SLA.0000000000003924

6. https://www.lequotidien.com/actualites/covid-19/les-chirurgiesnon-urgentes-reportees-ddba0490f354b53fdac3ccdd67561d4d

7. Aminian A, Safari S, Razeghian-Jahromi A et al (2020) COVID-19 outbreak and surgical practice: unexpected fatality in perioperative 
period. Ann Surg Publish Ahead of Print 272:e27-e29. https://doi. org/10.1097/SLA.0000000000003925

8. American College of Surgeons releases recommendations for surgical management of elective operations during COVID-19 pandemic

9. Hospital emergency data related to the COVID-19 epidemy in France

10. Owens WD, Felts JA, Spitznagel EL (1978) ASA physical status classifications: a study of consistency of ratings. Anesthesiology 49:239-243

11. Dindo D, Demartines N, Clavien P-A (2004) Classification of surgical complications: a new proposal with evaluation in a cohort of 6336 patients and results of a survey. Annals of Surgery 240:205213. https://doi.org/10.1097/01.sla.0000133083.54934.ae

12. Collard MK, Lefèvre JH, Batteux F, et al (2020) COVID-19 heath crisis: less colorectal resections and yet no more peritonitis or bowel obstruction as a collateral effect? Colorectal Dis codi.15199. https:// doi.org/10.1111/codi.15199

13. COVIDSurg Collaborative (2020) Global guidance for surgical care during the COVID-19 pandemic: Surgical care during the COVID-19 pandemic. Br J Surg. 107:1097-1103. https://doi.org/ $10.1002 /$ bjs. 11646

14. Thornton J (2020) Covid-19: A\&E visits in England fall by $25 \%$ in week after lockdown. BMJ m1401. https://doi.org/10.1136/bmj. m1401

15. Patriti A, Eugeni E, Guerra F (2020) What happened to surgical emergencies in the era of COVID-19 outbreak? Considerations of surgeons working in an Italian COVID-19 red zone. Updates Surg. 72:309-310. https://doi.org/10.1007/s13304-020-00779-6
16. Pan $\mathrm{L}, \mathrm{Mu} \mathrm{M}$, Yang $\mathrm{P}$ et al (2020) Clinical characteristics of COVID-19 patients with digestive symptoms in Hubei, China: a descriptive, cross-sectional, multicenter study. Am J Gastroenterol 115:766-773. https://doi.org/10.14309/ajg.0000000000000620

17. Wang D, Hu B, Hu C et al (2020) Clinical characteristics of 138 hospitalized patients with 2019 novel coronavirus-infected pneumonia in Wuhan, China. JAMA 323:1061-1069. https://doi.org/10. 1001/jama.2020.1585

18. Chen Y, Chen L, Deng Q, et al (2020) The presence of SARS-CoV2 RNA in the feces of COVID-19 patients. J Med Virol jmv.25825. https://doi.org/10.1002/jmv.25825

19. Gu J, Han B, Wang J (2020) COVID-19: Gastrointestinal manifestations and potential fecal-oral transmission. Gastroenterology 158: 1518-1519. https://doi.org/10.1053/j.gastro.2020.02.054

20. The Lancet (2020) COVID-19: protecting health-care workers. The Lancet 395:922. https://doi.org/10.1016/S0140-6736(20)30644-9

21. Di Saverio S, Khan M, Pata F et al (2020) Laparoscopy at all costs? Not now during COVID-19 and not for acute care surgery and emergency colorectal surgery: a practical algorithm from a Hub Tertiary teaching hospital in Northern Lombardy, Italy. J Trauma Acute Care Surg 1:715-718. https://doi.org/10.1097/TA. 0000000000002727

22. Vigneswaran Y, Prachand VN, Posner MC et al (2020) What is the appropriate use of laparoscopy over open procedures in the current COVID-19 Climate? J Gastrointest Surg. 24:1686-1691. https:// doi.org/10.1007/s11605-020-04592-9

Publisher's note Springer Nature remains neutral with regard to jurisdictional claims in published maps and institutional affiliations. 